\title{
Explicit Exact and Third-Order-Accurate Pressure-Deflection Solutions for Oblique Shock and Expansion Waves
}

\author{
Dan Mateescu ${ }^{*}$
}

Aerospace Program, Mechanical Engineering Department, McGill University, Montreal, QC, Canada

\begin{abstract}
This paper presents explicit analytical solutions of the pressure coefficient and the pressure ratio across the oblique shock and expansion waves in function of the flow deflection angle. These new explicit pressure-deflection solutions can be efficiently used in solving applied aerodynamic problems in supersonic flows, such as the aerodynamics of airfoils and wings in supersonic-hypersonic flows and the shock and expansion waves interactions, and can be also used to increase the computational efficiency of the numerical methods based on the Riemann problem solution requiring the pressure-deflection solution of the oblique shock and expansion waves, such as the Godunov method.
\end{abstract}

Keywords: Shock waves, Prandtl-Meyer expansions, supersonic flows, aerodynamics.

\section{INTRODUCTION}

The solution of many applied aerodynamic problems in supersonic flows often requires explicit solutions of the pressure ratio, or the pressure coefficient, in function of the flow deflection angle for the oblique shock and expansion waves. Also, several numerical methods based on the solution of the Riemann problem, such as the Godunov method, require the pressure-deflection solution of the oblique shock and expansion waves, which are usually obtained by an iterative procedure (see for example Mateescu [1] and Loh \& Hui [2] ). In the absence of explicit analytical solutions, the solutions of the oblique shock and expansion waves are obtained from diagrams and tables (see for example Anderson [3-5], Saad [6], Yahya [7] and Carafoli, Mateescu and Nastase [8]), or numerically by solving iteratively the implicit equations.

However, exact analytical solutions in explicit pressuredeflection form, or eventually explicit third-order accurate solutions, would be more efficient for solving applied aerodynamic problems in supersonic-hypersonic flows, such as the shock and expansion waves interactions and the aerodynamics of airfoils and wings in supersonic-hypersonic flows, or to be efficiently used in the numerical methods which require the pressure-deflection solutions of the oblique shock and expansion waves (such as the Godunov method).

The aim of this paper is to obtain rigorous analytical solutions of the pressure coefficient and pressure ratio across the oblique shock waves in explicit form in function of the flow deflection angle. As a by-product, unitary third-order accurate solutions in explicit pressure-deflection form are also derived for both the oblique shocks and expansion waves.

*Address correspondence to this author at the Aerospace Program, Mechanical Engineering Department, McGill University, Montreal, QC, Canada; Tel: 514 398-6284; E-mail: dan.mateescu@mcgill.ca

\section{EXPLICIT EXACT ANALYTICAL SOLUTIONS FOR OBLIQUE SHOCK WAVES}

The conservation equations of continuity, momentum (normal and tangent to the shock) and energy for a thin shock wave are

$$
\begin{aligned}
& \rho_{1} V_{1 n}=\rho_{2} V_{2 n} \\
& p_{1}+\rho_{1} V_{1 n}^{2}=p_{2}+\rho_{2} V_{2 n}^{2} \\
& V_{1 t}=V_{2 t} \\
& \frac{1}{2} V_{1}^{2}+\frac{\gamma}{\gamma-1} \frac{p_{1}}{\rho_{1}}=\frac{1}{2} V_{2}^{2}+\frac{\gamma}{\gamma-1} \frac{p_{2}}{\rho_{2}}
\end{aligned}
$$

where $V_{1}, p_{1}, \rho_{1}$, and $V_{2}, p_{2}, \rho_{2}$, are the fluid velocity, pressure and density before and after the shock, and $V_{1 n}$, $V_{1 t}$ and $V_{2 n}, V_{2 t}$ are the velocity components normal and tangent to the shock defined as

$$
\begin{aligned}
& V_{1 n}=V_{1} \sin \beta, V_{1 t}=V_{1} \cos \beta, V_{1 n}^{2}+V_{1 t}^{2}=V_{1}^{2} \\
& V_{2 n}=V_{2} \sin (\beta-\tau), V_{2 t}=V_{2} \cos (\beta-\tau), V_{2 n}^{2}+V_{2 t}^{2}=V_{2}^{2}
\end{aligned}
$$

where $\beta$ and $\tau$ are the shock inclination angle and the flow deflection angle behind the shock with respect to the upstream flow direction (Fig. 1).

The Mach numbers before and after the shock are defined using the speeds of sound $a_{1}=\sqrt{\gamma p_{1} / \rho_{1}}$ and $a_{2}=\sqrt{\gamma p_{2} / \rho_{2}}$ as

$M_{1}=V_{1} / a_{1}, M_{2}=V_{2} / a_{2}, M_{1 n}=V_{1 n} / a_{1}=M_{1} \sin \beta$

The system of equations (1) can be reduced to the quadratic equation of the density ratio $\eta=\rho_{1} / \rho_{2}=V_{2 n} / V_{1 n}$ in the form 
$(\eta-1)\left[\eta-\left(\gamma-1+2 / M_{1 n}^{2}\right) /(\gamma+1)\right]=0$

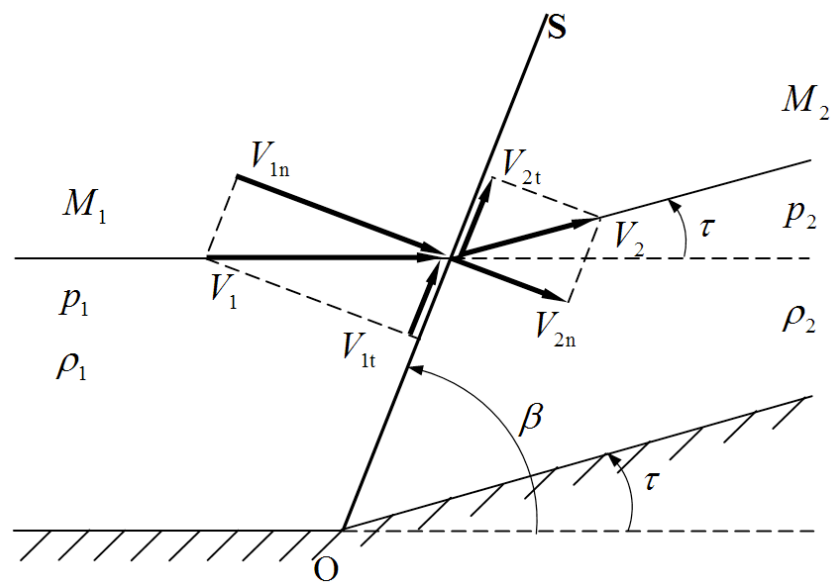

Fig. (1). Shock wave geometry.

with the solution (discounting the trivial solution $\eta=1$ )

$\eta=\frac{V_{2 n}}{V_{1 n}}=\frac{\rho_{1}}{\rho_{2}}=\frac{2}{\gamma+1} \frac{1}{M_{1}^{2} \sin ^{2} \beta}+\frac{\gamma-1}{\gamma+1}$

$\frac{p_{2}}{p_{1}}=\frac{2 \gamma}{\gamma+1} M_{1}^{2} \sin ^{2} \beta-\frac{\gamma-1}{\gamma+1}$

The unknown shock angle $\beta$ is defined by the boundary condition behind the shock

$\tan (\beta-\tau)=\left(\frac{2}{\gamma+1} \frac{1}{M_{1}^{2} \sin ^{2} \beta}+\frac{\gamma-1}{\gamma+1}\right) \tan \beta$

which can also be expressed as

$\sin \tau=\frac{(1-\eta) \sin \beta}{\sqrt{1-\left(1-\eta^{2}\right) \sin ^{2} \beta}} \cos \beta$

These are not explicit equations of $\beta$ in function of $\tau$ and the solution is usually obtained from diagrams and tables [3-7], or numerically by an iterative procedure.

By defining the pressure coefficient for the flow behind the shock as

$C_{p}=\left(\frac{p_{2}}{p_{1}}-1\right) \frac{2}{\gamma M_{1}^{2}} \Rightarrow C_{p}=\frac{4}{\gamma+1}\left(\sin ^{2} \beta-\frac{1}{M_{1}^{2}}\right)$

the boundary condition (6b) can be expressed in the form

$\sin \tau=\frac{1}{2} C_{p} \frac{\sqrt{4 M_{1}^{2}-4-(\gamma+1) M_{1}^{2} C_{p}}}{\sqrt{4+(\gamma+1) M_{1}^{2} C_{p}-C_{p}\left(4+\gamma M_{1}^{2} C_{p}\right)}}$

This can be recast as a cubic equation in terms of the pressure coefficient $C_{p}$

$C_{p}^{3}-3 b C_{p}^{2}-4 \sin ^{2} \tau\left(\frac{4}{(\gamma+1) M_{1}^{2}}-1\right) C_{p}+\frac{16 \sin ^{2} \tau}{(\gamma+1) M_{1}^{2}}=0$

with the solutions

$$
\begin{aligned}
& C_{p}=b\left[1-2 \sqrt{q} \cos \frac{\pi+\alpha}{3}\right] \\
& C_{p}^{S}=b\left[1+2 \sqrt{q} \cos \frac{\alpha}{3}\right] \\
& C_{p}^{E}=b\left[1-2 \sqrt{q} \cos \frac{\pi-\alpha}{3}\right]
\end{aligned}
$$

where

$$
\begin{aligned}
& b=\frac{4}{3(\gamma+1)}\left(1-\frac{1}{M_{1}^{2}}+\gamma \sin ^{2} \tau\right) \\
& \alpha=\cos ^{-1} \frac{r}{\sqrt{q^{3}}} \\
& q=1-\frac{4 \sin ^{2} \tau}{3 b^{2}}\left(1-\frac{4}{\gamma+1} \frac{1}{M_{1}^{2}}\right) \\
& r=\frac{3 q-1}{2}-\frac{8 \sin ^{2} \tau}{(\gamma+1) b^{3} M_{1}^{2}}
\end{aligned}
$$

Equations (10) and (11) represent the exact explicit solutions for the weak shock waves (which is the main aim of this paper) and, respectively, for the strong shock waves, occurring in special situations. These two solutions become equal for $\alpha=\pi$, which represents the limit case of the attached oblique shocks, just before the shock wave detachment.

Equation (12) represents the solution for an expansion shock, which is associated with an unphysical decrease of entropy in adiabatic flow. This entropy decrease is however very small for a certain range of values of the deflection angle $\tau$ and Mach number $M_{1}$, in which case equation (12) provide third-order accurate solutions of the Prandtl-Meyer expansions. This expansion-shock solution has however an unphysical upper limit of the deflection angle, which corresponds to the limit angle at the shock-wave detachment, restricting thus its validity to a narrower range of deflection angles for various upstream Mach numbers.

The exact changes of the pressure, density, speed of sound and Mach number across the shock and the shock angle $\beta$ can be calculated from the exact explicit solution of the pressure coefficient (10) for weak shocks, or from equation (11) for strong shocks, as

$$
\begin{aligned}
& \frac{p_{2}}{p_{1}}=1+\frac{\gamma}{2} M_{1}^{2} C_{p} \Rightarrow \frac{p_{2}}{p_{1}}=1+\frac{\gamma}{2} M_{1}^{2} b\left[1-2 \sqrt{q} \cos \frac{\pi+\alpha}{3}\right] \\
& \frac{\rho_{1}}{\rho_{2}}=\frac{4+(\gamma-1) M_{1}^{2} C_{p}}{4+(\gamma+1) M_{1}^{2} C_{p}}=\frac{V_{2 n}}{V_{1 n}} \\
& \frac{a_{2}}{a_{1}}=\sqrt{\frac{p_{2}}{p_{1}} \frac{\rho_{1}}{\rho_{2}}}=\sqrt{1+\frac{\gamma}{2} M_{1}^{2} C_{p}} \sqrt{\frac{4+(\gamma-1) M_{1}^{2} C_{p}}{4+(\gamma+1) M_{1}^{2} C_{p}}} \\
& \frac{M_{2}}{M_{1}}=\sqrt{\frac{4+(\gamma-1) M_{1}^{2} C_{p}}{4+2 \gamma M_{1}^{2} C_{p}}} \frac{2}{\sqrt{4+(\gamma+1) M_{1}^{2} C_{p}} \cos \tau-\sqrt{4 M_{1}^{2}-4-(\gamma+1) M_{1}^{2} C_{p}} \sin \tau}
\end{aligned}
$$




$$
\begin{aligned}
& \frac{V_{2}}{V_{1}}=\frac{1}{\sqrt{4+(\gamma+1) M_{1}^{2} C_{p}}} \frac{4+(\gamma-1) M_{1}^{2} C_{p}}{\sqrt{4+(\gamma+1) M_{1}^{2} C_{p}} \cos \tau-\sqrt{4 M_{1}^{2}-4-(\gamma+1) M_{1}^{2} C_{p}} \sin \tau} \\
& \sin \beta=\frac{1}{M_{1}} \sqrt{1+\frac{\gamma+1}{4} M_{1}^{2} C_{p}}
\end{aligned}
$$

Thus, equations (10) and (11) represent, together with (14a-f), the exact analytical solutions in explicit pressuredeflection form of the weak and strong shock waves.

\section{EXPLICIT THIRD-ORDER ACCURATE SOLUTIONS FOR OBLIQUE SHOCK AND EXPANSION WAVES}

The boundary condition (8) can also be expressed as

$\sqrt{1+\frac{\gamma+1}{4} M_{1}^{2} C_{p}} \sin \tau=\frac{B}{2 \sqrt{\kappa}} C_{p}$

where $B$ is defined as

$B=\sqrt{M_{1}^{2}-1}$

and where the parameter $\kappa$, which has values close to unity, is defined as

$\kappa=\left(1-C_{p} \frac{4+\gamma M_{1}^{2} C_{p}}{4+(\gamma+1) M_{1}^{2} C_{p}}\right) \frac{4}{4-(\gamma+1) M_{1}^{2} C_{p} / B^{2}}$

Equation (15) can be thus recast formally as a quadratic equation,

$$
\left(\frac{B}{2 \sin \tau} C_{p}\right)^{2}-2 \kappa K\left(\frac{B}{2 \sin \tau} C_{p}\right)-\kappa=0
$$

with two solutions for the positive and negative values of $\tau$ expressed as

$$
C_{p}=\frac{2 \sin \tau}{B}\left[\sqrt{\kappa+(\kappa K)^{2}}+\kappa K\right]
$$

where $K$ represents a similarity parameter for supersonichypersonic flows

$$
K=\frac{2 \sin \tau}{B} \frac{\gamma+1}{8} M_{1}^{2}
$$

For $\kappa=1$ one obtains the second-order solution [8]

$$
C_{p C}=\frac{2 \sin \tau}{B}\left[\sqrt{1+K^{2}}+K\right]
$$

Considering for $\kappa$ the value based on $C_{p C}$

$\kappa_{0}=\left(1-C_{p C} \frac{4+\gamma M_{1}^{2} C_{p C}}{4+(\gamma+1) M_{1}^{2} C_{p C}}\right) \frac{4}{4-(\gamma+1) M_{1}^{2} C_{p C} / B^{2}}$

one obtains the present third-order solution

$$
C_{p}^{M}=\frac{2 \sin \tau}{B}\left[\sqrt{\kappa_{0}+\left(\kappa_{0} K\right)^{2}}+\kappa_{0} K\right]
$$

$$
\begin{aligned}
& \frac{p_{2}}{p_{1}}=1+\frac{\gamma}{2} M_{1}^{2} C_{p}^{M} \quad \Rightarrow \\
& \quad \frac{p_{2}}{p_{1}}=1+\gamma M_{1}^{2} \frac{\sin \tau}{B}\left[\sqrt{\kappa_{0}+\left(\kappa_{0} K\right)^{2}}+\kappa_{0} K\right]
\end{aligned}
$$

with the other flow variables behind the shock defined by equations (14b-f).

Equations (23) and (24) represent the third-order unitary solutions in explicit pressure-deflection form for both the oblique shocks (for $\tau>0$ ) and Prandtl-Meyer expansions (for $\tau<0$ ), since the unphysical entropy decrease associated with the third-order solution (23) of an expansion shock is negligibly small for a certain range of Mach numbers and deflection angles. The other flow variables after the expansion are calculated more accurately from (24) by using the isentropic relations, instead of $(14 \mathrm{~b}-\mathrm{e})$, in order to eliminate the effect of the unphysical entropy variation, in the form

$\frac{\rho_{2}}{\rho_{1}}=\left(\frac{p_{2}}{p_{1}}\right)^{1 / \gamma}$

$\frac{a_{2}^{2}}{a_{1}^{2}}=\frac{p_{2}}{p_{1}} \frac{\rho_{1}}{\rho_{2}}=\left(\frac{p_{2}}{p_{1}}\right)^{1-1 / \gamma}$

$M_{2}^{2}=\left(\frac{2}{\gamma-1}+M_{1}^{2}\right)\left(\frac{p_{1}}{p_{2}}\right)^{1-1 / \gamma}-\frac{2}{\gamma-1}$

$\frac{V_{2}}{V_{1}}=\frac{M_{2}}{M_{1}} \frac{a_{2}}{a_{1}}$

The values of the supersonic-hypersonic similarity parameter $K=\frac{2 \sin \tau}{B} \frac{\gamma+1}{8} M_{1}^{2}$ define the following flow regimes:

(i) Linear supersonic flows, for $K<<1$ and $M_{1} \leq 3$, when equation (23) reduces to the linear solution $C_{p}=2 \sin \tau / B$ (or $C_{p} \approx 2 \tau / B$ with $\tau$ in radians), which is valid for both compressions $(\tau>0)$ and expansions $(\tau<0)$.

(ii) Supersonic-moderate hypersonic flows, for $K \leq 1$.

(iii) Hypersonic flows, for $K>1$.

The pair of explicit solutions (10) and (12) represent also solutions for compressions and expansions, solving rigorously the oblique shocks $(\tau>0)$ and, respectively, with third-order accuracy the expansion waves $(\tau<0)$.

\section{NUMERICAL VALIDATIONS}

The present explicit exact solutions of the pressure coefficient $C_{p}$ in function of the deflection angle $\tau$ for the weak shock waves (10) and for the strong shocks (11) were

which leads to the pressure ratio across the shock. 


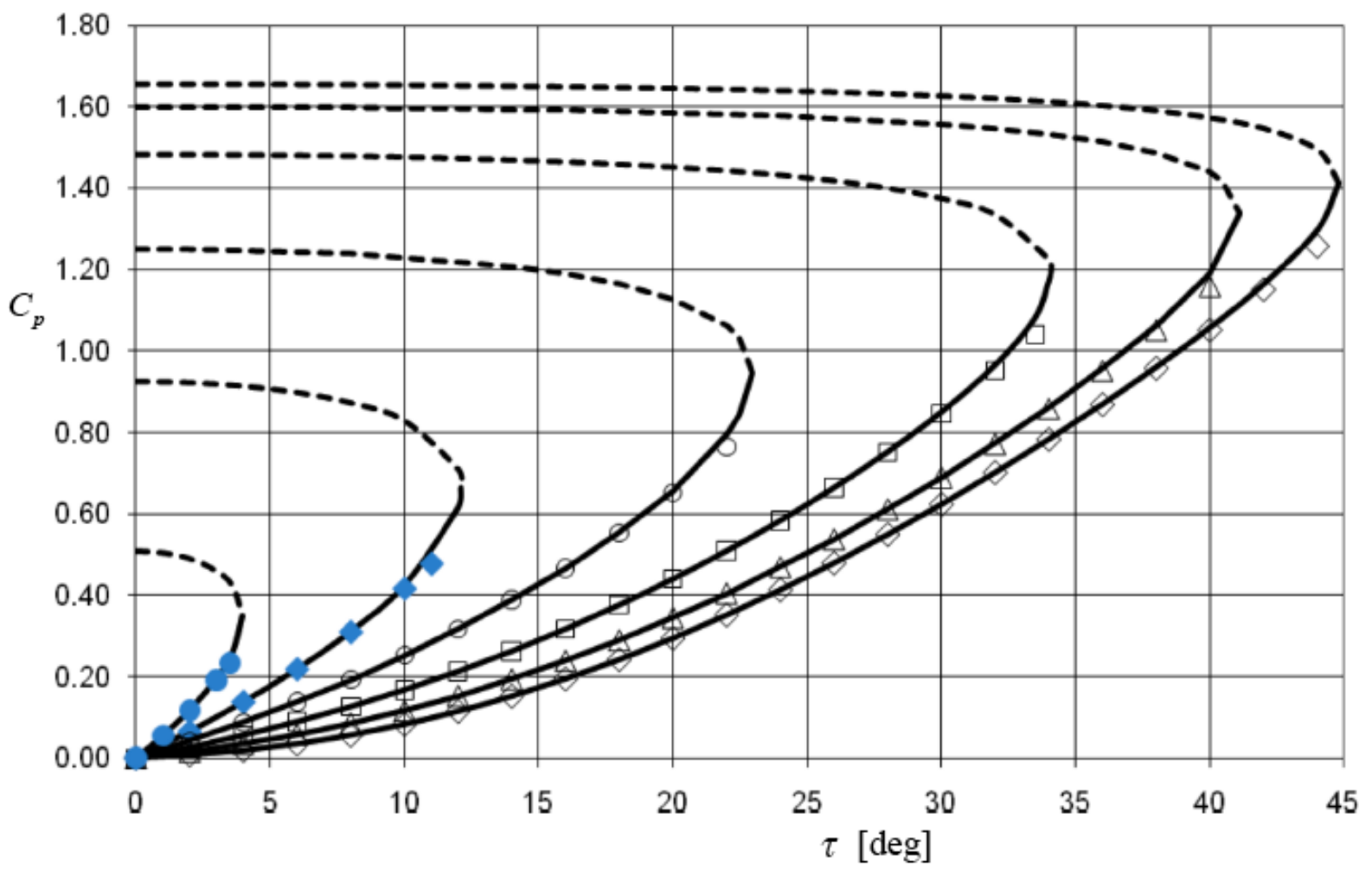

Fig. (2). Oblique shock waves: Variation of the pressure coefficient $C_{p}$ with the deflection angle $\tau$ for various values of the upstream Mach number $M_{1}$. Comparison between:

Present unitary explicit third-order-accurate solutions $C_{p}^{M}$, defined by eq. (23) for $\tau>0: \bullet M_{1}=1.2 ; \bullet M_{1}=1.5 ; \circ M_{1}=2.0$; $\square M_{1}=3.0$; $\Delta M_{1}=5.0 ; \diamond M_{1}=12$. — Present exact weak-shock solutions $C_{p}$ defined by eq. (10) in explicit form. ------ Present exact strong-shock solutions $C_{p}^{S}$ defined by eq. (11) in explicit form.

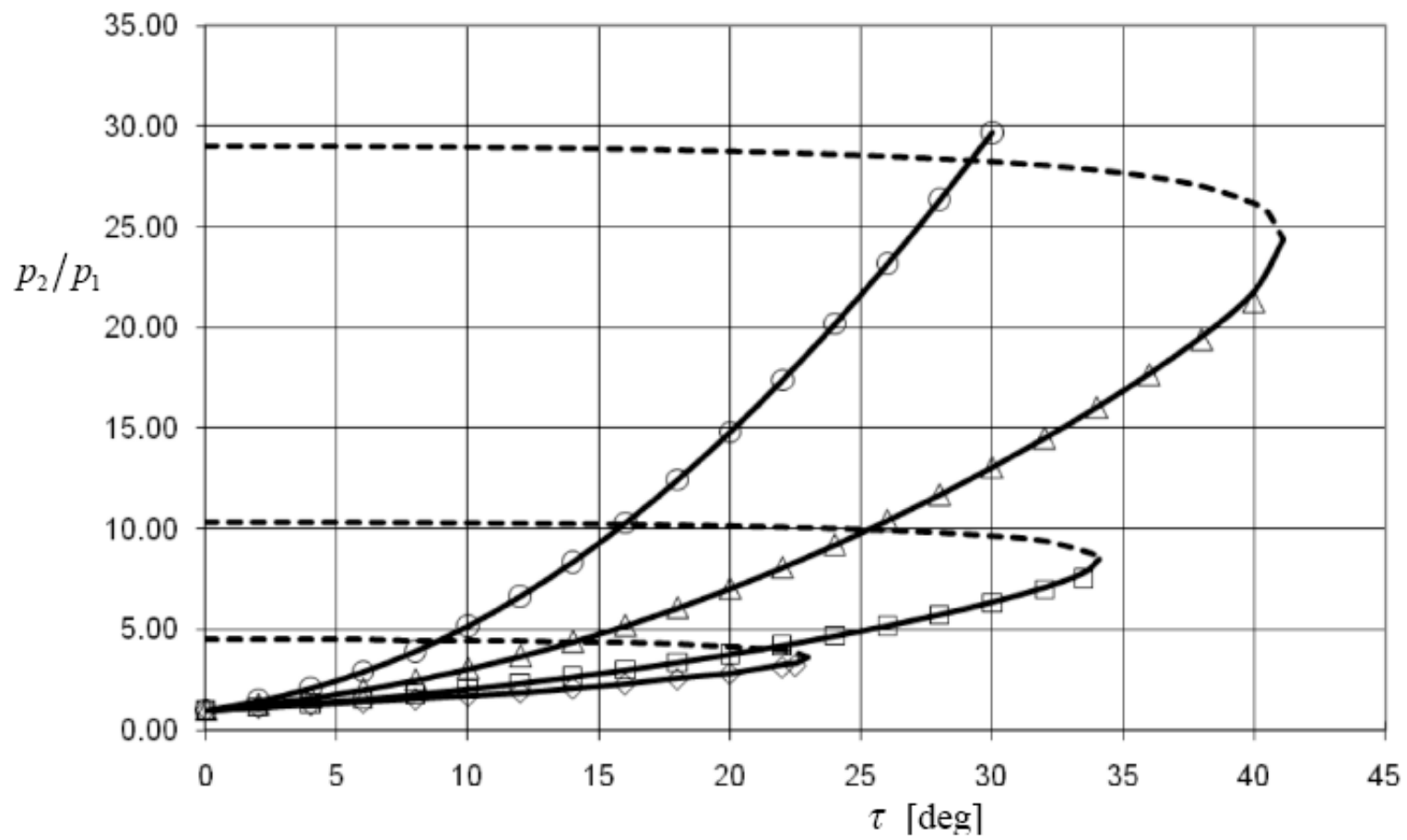

Fig. (3). Oblique shock waves: Variation of the pressure ratio $p_{2} / p_{1}$ with the deflection angle $\tau$ for various values of the upstream Mach number $M_{1}$. Comparison between: Present unitary explicit third-order-accurate solutions, defined by eq. (24) for $\tau>0: \diamond M_{1}=2$; $\square M_{1}=3.0$; $\Delta M_{1}=5.0 ; \circ M_{1}=8.0$. — Present exact weak-shock solutions defined by equations (10), (14a) in explicit form. ------ Present exact strongshock solutions defined by equations (11), (14a) in explicit form. 


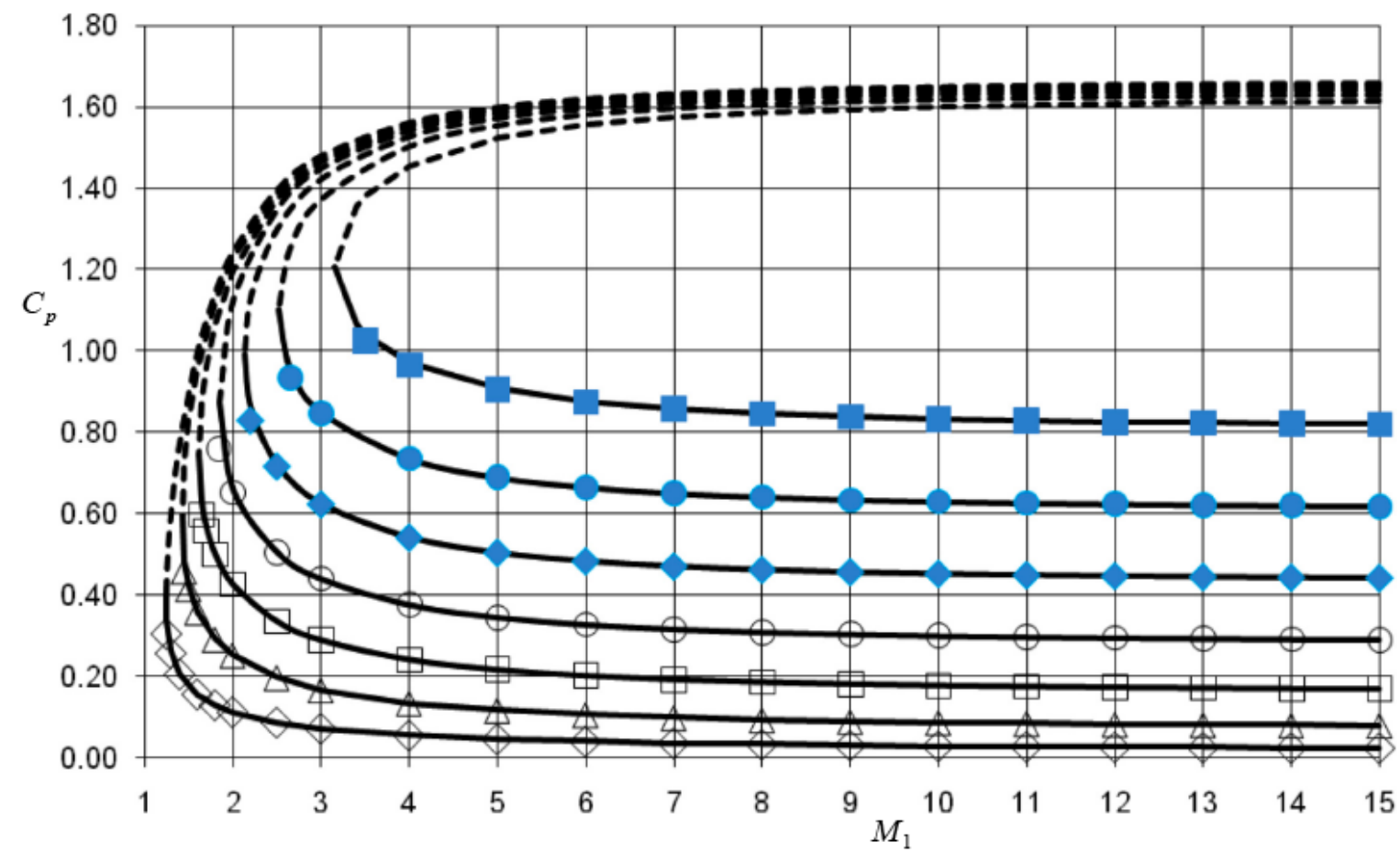

Fig. (4). Oblique shock waves: Variation of the pressure coefficient $C_{p}$ with the upstream Mach number $M_{1}$ for various values of the deflection angle $\tau$. Comparison between: Present unitary explicit third-order-accurate solutions $C_{p}^{M}$, defined by eq. (23) for $\tau>0$ : $\diamond \tau=5^{\circ} ; \Delta \tau=10^{\circ} ; \square \tau=15^{\circ} ; \circ \tau=20^{\circ} ; \diamond \tau=25^{\circ} ; \bullet \tau=30^{\circ} ; \square \tau=35^{\circ}$. — Present exact weak-shock solutions $C_{p}$ defined by eq. (10) in explicit form. ------- Present exact strong-shock solutions $C_{p}^{S}$ defined by eq. (11) in explicit form.

Table 1. Oblique shock wave: Relative errors of the present third-order-accurate solutions of the pressure coefficient $C_{p}^{M}$, calculated with the unitary shock-expansion formula (23) for $\tau>0$, with respect to the exact solutions, for various values of the flow deflection angle $\tau$ and upstream Mach number $M_{1}$

\begin{tabular}{|c|c|c|c|c|c|c|c|c|}
\hline$\tau$ & $M_{1}=1.2$ & $M_{1}=1.5$ & $M_{1}=2$ & $M_{1}=\mathbf{3}$ & $M_{1}=5$ & $M_{1}=8$ & $M_{1}=12$ & $M_{1}=15$ \\
\hline $2^{\circ}$ & $-0.67 \%$ & $0.00 \%$ & $0.00 \%$ & $0.00 \%$ & $0.00 \%$ & $0.00 \%$ & $0.00 \%$ & $0.00 \%$ \\
\hline $4^{\circ}$ & - - - & $0.00 \%$ & $0.00 \%$ & $0.00 \%$ & $0.00 \%$ & $0.00 \%$ & $0.00 \%$ & $0.00 \%$ \\
\hline $6^{\circ}$ & $\ldots$ & $-0.02 \%$ & $0.00 \%$ & $-0.01 \%$ & $0.00 \%$ & $0.00 \%$ & $0.00 \%$ & $0.00 \%$ \\
\hline $8^{\circ}$ & -- & $-0.12 \%$ & $0.00 \%$ & $-0.01 \%$ & $0.00 \%$ & $0.00 \%$ & $0.00 \%$ & $0.00 \%$ \\
\hline $10^{\circ}$ & -- & $-0.49 \%$ & $0.01 \%$ & $-0.01 \%$ & $0.00 \%$ & $0.00 \%$ & $0.00 \%$ & $0.00 \%$ \\
\hline $14^{\circ}$ & - - - & - - - & $0.00 \%$ & $0.00 \%$ & $0.00 \%$ & $0.00 \%$ & $0.00 \%$ & $0.00 \%$ \\
\hline $16^{\circ}$ &.- & $\ldots$ & $-0.07 \%$ & $0.01 \%$ & $0.00 \%$ & $0.00 \%$ & $0.00 \%$ & $0.00 \%$ \\
\hline $18^{\circ}$ & $\ldots$ & $\ldots$ & $-0.29 \%$ & $0.02 \%$ & $0.01 \%$ & $0.00 \%$ & $0.00 \%$ & $0.00 \%$ \\
\hline $20^{\circ}$ & -- & $\ldots$ & $-0.97 \%$ & $0.03 \%$ & $0.01 \%$ & $0.00 \%$ & $0.00 \%$ & $0.00 \%$ \\
\hline $22^{\circ}$ & $-\ldots$ & $-\cdots$ & $-3.57 \%$ & $0.04 \%$ & $0.01 \%$ & $0.00 \%$ & $0.00 \%$ & $0.00 \%$ \\
\hline $30^{\circ}$ & -- & $\ldots$ & $-\ldots$ & $-0.45 \%$ & $-0.03 \%$ & $-0.03 \%$ & $-0.04 \%$ & $-0.04 \%$ \\
\hline $32^{\circ}$ & - - & $\ldots$ & - - & $-1.41 \%$ & $-0.07 \%$ & $-0.06 \%$ & $-0.06 \%$ & $-0.06 \%$ \\
\hline $34^{\circ}$ & - - & $\ldots$ & - - & $\ldots$ & $-0.18 \%$ & $-0.11 \%$ & $-0.10 \%$ & $-0.10 \%$ \\
\hline $36^{\circ}$ & -- & -- & -- & -- & $-0.39 \%$ & $-0.20 \%$ & $-0.18 \%$ & $-0.17 \%$ \\
\hline $38^{\circ}$ & $-\ldots$ & $\ldots$ & $-\cdots$ & $-\cdots$ & $-0.91 \%$ & $-0.39 \%$ & $-0.31 \%$ & $-0.29 \%$ \\
\hline $40^{\circ}$ & $\ldots$ & $-\ldots$ & $\ldots$ & $\ldots$ & $-2.52 \%$ & $-0.77 \%$ & $-0.58 \%$ & $-0.54 \%$ \\
\hline
\end{tabular}




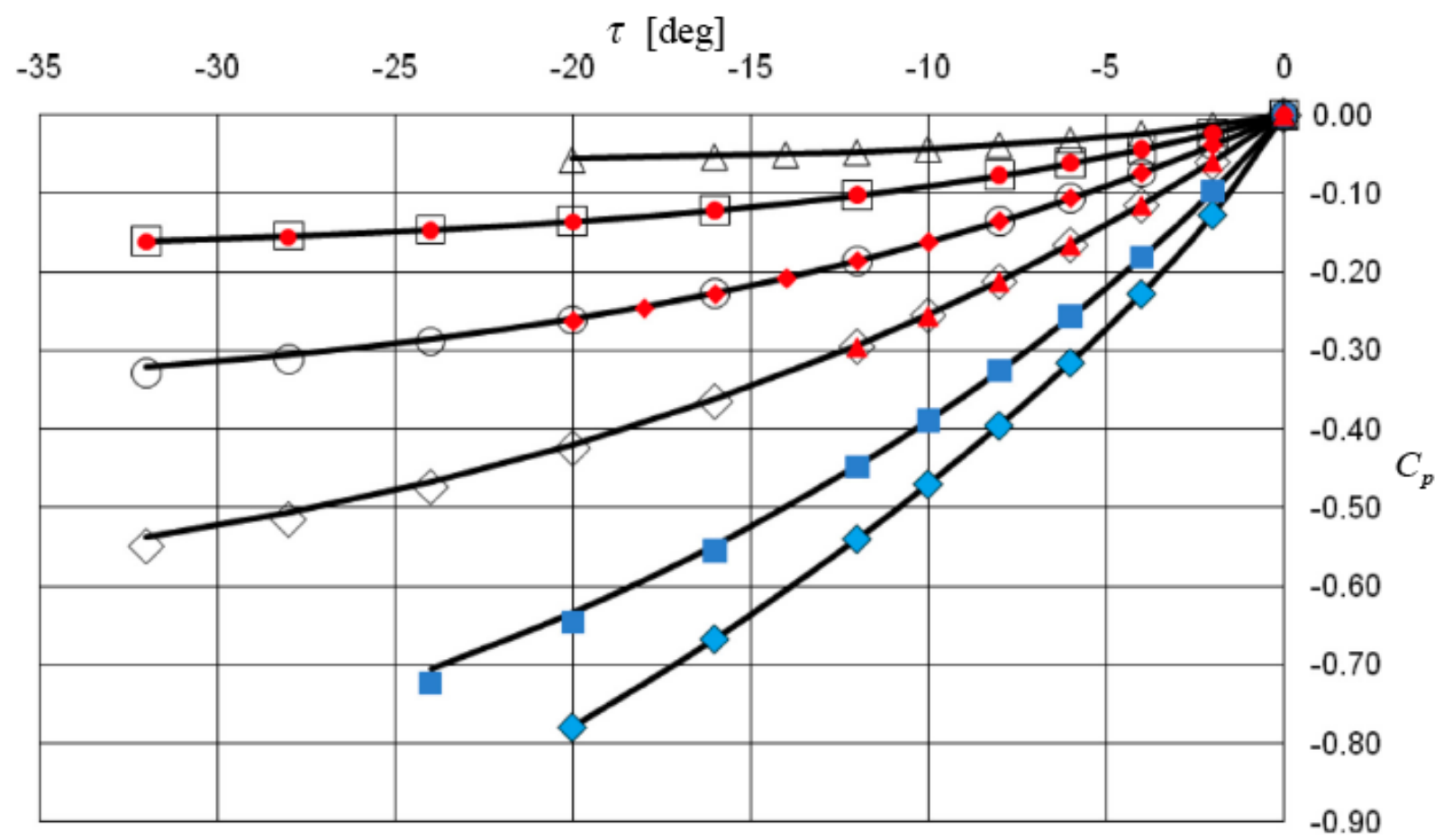

Fig. (5). Expansion waves: Variation of the pressure coefficient $C_{p}$ with the deflection angle $\tau$ for various values of the upstream Mach number $M_{1}$. Comparison between: Present unitary explicit third-order-accurate solutions $C_{p}^{M}$, defined by eq. (23) for $\tau<0: \diamond M_{1}=1.1$; $M_{1}=1.2 ; \diamond M_{1}=1.5 ; \circ M_{1}=2.0 ; \square M_{1}=3.0 ; \Delta M_{1}=5.0$. Present expansion-shock solutions $C_{p}^{E}$, defined by eq. (12), for: $\Delta M_{1}=1.5 ; \diamond M_{1}=2.0$; - $M_{1}=3.0$. P Prandtl-Meyer expansion: numerical solutions (based on Newton's iterative method).

found in perfect agreement with the classical indirect solutions (which calculate the deflection angle $\tau$ for a specified inclination angle $\beta$ of the oblique shock wave) and with the numerical solutions obtained by solving iteratively the implicit equations (6a or b) and (5a), which are given in diagram and table form in References [3-7].

The present unitary third-order solutions for compression-expansion (23), and the expansion-shock solution (12), are validated in Figs. (2-4) and Table 1 by comparison with the exact solutions for the case of oblique shock waves ( $\tau>0$ ), and in Fig. (5) and Tables 2 and $\mathbf{3}$ for the case of expansion waves $(\tau<0)$ by comparison with Prandtl-Meyer solutions computed numerically by using Newton's iterative procedure (based on the derivative of the Prandtl-Meyer function $v(M)$ with respect to the Mach number).

The variations of the pressure coefficient, $C_{p}$, and of the pressure ratio across the shock, $p_{2} / p_{1}$, with the deflection angle $\tau$ are illustrated in Figs. $(2,3)$ for various values of the upstream Mach number $M_{1}$. Similarly, Fig. (4) illustrates the variations of the pressure coefficient $C_{p}$ with the upstream Mach number $M_{1}$ for various values of the deflection angle $\tau$. One can notice from these figures that the present unitary third-order solutions $C_{p}^{M}$, defined by equation (23) for $\tau>0$, are in excellent agreement with the exact solutions for a wide range of transonic, supersonic and hypersonic upstream Mach numbers, $M_{1}$, between 1.1 and 15 (and even higher) and for a wide range of flow deflection angles $\tau$, up to $40^{\circ}$ (or more) in function of $M_{1}$.

The relative differences between the present unitary third-order solutions $C_{p}^{M}$ for $\tau>0$ and the exact weakshock solutions are shown in Table 1. One can notice that the present explicit third-order solution $C_{p}^{M}$ has an excellent accuracy, with relative errors less than $1 \%$ for a wide range of Mach numbers and flow deflection angles, except very near to the detached shock conditions where the errors are somewhat larger.

For the expansion case, the present unitary third-order solutions $C_{p}^{M}$ defined by equation (23) for $\tau<0$, and the expansion-shock solutions $C_{p}^{E}$, defined by equation (12), are compared with the Prandtl-Meyer solutions (computed numerically using Newton's iterative method) in Fig. (5) and Tables $\mathbf{2}$ and $\mathbf{3}$. One can notice that the present unitary thirdorder solution $C_{p}^{M}$ for $\tau<0$, and the present expansionshock solutions $C_{p}^{E}$, are in very good agreement with the numerical solutions of the Prandtl-Meyer expansion for a wide range of upstream Mach numbers $M_{1}$ and flow deflection angles $\tau$. 
Table 2. Expansion wave: Relative errors of the present third-order-accurate solutions of the pressure coefficient $C_{p}^{M}$, calculated with the unitary compression-expansion formula (23) for $\tau<0$, with respect to the numerical solutions of Prandtl-Meyer expansions, for various values of the flow deflection angle $\tau$ and upstream Mach number $M_{1}$

\begin{tabular}{|c|c|c|c|c|c|c|c|}
\hline $\boldsymbol{\tau}$ & $\boldsymbol{M}_{\mathbf{1}}=\mathbf{1 . 2}$ & $\boldsymbol{M}_{\mathbf{1}}=\mathbf{1 . 5}$ & $\boldsymbol{M}_{\mathbf{1}}=\mathbf{2}$ & $\boldsymbol{M}_{\mathbf{1}}=\mathbf{3}$ & $\boldsymbol{M}_{\mathbf{1}}=\mathbf{5}$ & $\boldsymbol{M}_{\mathbf{1}}=\mathbf{8}$ & $\boldsymbol{M}_{\mathbf{1}}=\mathbf{1 2}$ \\
\hline \hline$-2^{\circ}$ & $-0.12 \%$ & $0.02 \%$ & $0.01 \%$ & $-0.01 \%$ & $-0.03 \%$ & $-0.08 \%$ & $-0.14 \%$ \\
\hline$-4^{\circ}$ & $-0.21 \%$ & $0.07 \%$ & $0.03 \%$ & $-0.01 \%$ & $-0.08 \%$ & $-0.12 \%$ & $0.08 \%$ \\
\hline$-6^{\circ}$ & $-0.15 \%$ & $0.15 \%$ & $0.07 \%$ & $0.00 \%$ & $-0.06 \%$ & $0.14 \%$ & $1.11 \%$ \\
\hline$-8^{\circ}$ & $0.03 \%$ & $0.25 \%$ & $0.12 \%$ & $0.04 \%$ & $0.08 \%$ & $0.78 \%$ & \\
\hline$-10^{\circ}$ & $0.30 \%$ & $0.37 \%$ & $0.20 \%$ & $0.12 \%$ & $0.38 \%$ & & \\
\hline$-12^{\circ}$ & $0.62 \%$ & $0.51 \%$ & $0.29 \%$ & $0.26 \%$ & $0.83 \%$ & & \\
\hline$-16^{\circ}$ & $1.35 \%$ & $0.81 \%$ & $0.54 \%$ & $0.71 \%$ & & \\
\hline$-20^{\circ}$ & & $1.15 \%$ & $0.89 \%$ & $1.40 \%$ & & & \\
\hline$-24^{\circ}$ & & & $1.33 \%$ & & & \\
\hline
\end{tabular}

Table 3. Expansion wave: Relative errors of the present third-order solutions of the pressure coefficient $C_{p}^{E}$, calculated with the expansion-shock formula (12), with respect to the numerical solutions of Prandtl-Meyer expansions, for various values of the flow deflection angle $\tau$ and upstream Mach number $M_{1}$

\begin{tabular}{|c|c|c|c|c|c|c|c|}
\hline $\boldsymbol{\tau}$ & $\boldsymbol{M}_{\mathbf{1}}=\mathbf{1 . 2}$ & $\boldsymbol{M}_{\mathbf{1}}=\mathbf{1 . 5}$ & $\boldsymbol{M}_{\mathbf{1}}=\mathbf{2}$ & $\boldsymbol{M}_{\mathbf{1}}=\mathbf{3}$ & $\boldsymbol{M}_{\mathbf{1}}=\mathbf{5}$ & $\boldsymbol{M}_{\mathbf{1}}=\mathbf{8}$ & $\boldsymbol{M}_{\mathbf{1}}=\mathbf{1 2}$ \\
\hline \hline$-2^{\circ}$ & -0.01 & $0.02 \%$ & $0.01 \%$ & $0.00 \%$ & $-0.03 \%$ & $-0.08 \%$ & $-0.14 \%$ \\
\hline$-4^{\circ}$ & & $0.07 \%$ & $0.04 \%$ & $0.00 \%$ & $-0.07 \%$ & $-0.11 \%$ & $0.08 \%$ \\
\hline$-6^{\circ}$ & & $0.15 \%$ & $0.10 \%$ & $0.02 \%$ & $-0.04 \%$ & $0.15 \%$ & $1.12 \%$ \\
\hline$-8^{\circ}$ & & $0.26 \%$ & $0.18 \%$ & $0.09 \%$ & $0.11 \%$ & $0.79 \%$ & \\
\hline$-10^{\circ}$ & & $0.41 \%$ & $0.30 \%$ & $0.20 \%$ & $0.42 \%$ & & \\
\hline$-12^{\circ}$ & & $0.59 \%$ & $0.45 \%$ & $0.38 \%$ & $0.90 \%$ & & \\
\hline$-16^{\circ}$ & & & $0.89 \%$ & $0.96 \%$ & & & \\
\hline$-20^{\circ}$ & & & $1.49 \%$ & & & \\
\hline
\end{tabular}

The relative differences between the present unitary third-order solutions, $C_{p}^{M}$ for $\tau<0$, or the expansion-shock solutions, $C_{p}^{E}$, and the exact numerical solutions of PrandtlMeyer expansion are shown in Tables 2 and 3. One can notice that the present explicit third-order solutions $C_{p}^{M}$ and $C_{p}^{E}$ have a very good accuracy, with relative errors less than $1 \%$ for a wide range of Mach numbers and deflection angles.

However, $C_{p}^{M}$ has a better accuracy than $C_{p}^{E}$ over a wider range of deflection angles for various upstream Mach numbers. In addition, $C_{p}^{M}$ is defined by a simpler algebraic expression (23) and is a unitary compression-expansion solution valid for both the oblique shocks $(\tau>0)$ and Prandtl-Meyer expansions $(\tau<0)$.

For these reasons, $C_{p}^{M}$ represents a better choice as a unitary third-order accurate solution.

\section{CONCLUSIONS}

Explicit exact solutions of the pressure coefficient and the pressure ratio across the shock wave in function of the flow deflection angle $\tau$ are derived in this paper for both the weak (10) and strong (11) oblique shock waves in supersonic-hypersonic flows. These explicit exact solutions were found in perfect agreement with the classical indirect solutions (calculating the flow deflection angle $\tau$ for a specified inclination angle $\beta$ of the oblique shock wave) and with the numerical solutions obtained by solving iteratively the implicit equations, which are given in diagram and table form in References [3-7].

A unitary shock-expansion solution (23), denoted as $C_{p}^{M}$, is also derived in explicit pressure-deflection form, solving with third-order accuracy (less than $1 \%$ errors) both the oblique shocks $(\tau>0)$ and the expansion waves $(\tau<0)$ for a wide range of upstream Mach numbers and flow deflection angles. 
An explicit expansion-shock solution (12) in pressuredeflection form, denoted as $C_{p}^{E}$, was also derived as a thirdorder solution for the expansion waves, which also provides accurate results for a wide range of upstream Mach numbers. This solution has however an unphysical upper limit of the deflection angle, corresponding to the limit angle of the shock-wave detachment. Due to this limitation, the unitary shock-expansion solution $C_{p}^{M}$ is the best choice as the unitary third-order-accurate solution for both the shock $(\tau>0)$ and expansion $(\tau<0)$ waves for a wide range of supersonic and hypersonic Mach numbers and flow deflection angles.

These new explicit pressure-deflection solutions can be efficiently used in solving applied aerodynamic problems in supersonic flows, such as the aerodynamics of airfoils and wings in supersonic-hypersonic flows and the shock and expansion waves interactions, and can be also used to increase the computational efficiency of the numerical methods based on the Riemann problem solution, such as the Godunov method, which require the pressure-deflection solution of the oblique shock and expansion waves.

\section{ACKNOWLEDGEMENTS}

The support of the Natural Sciences and Engineering Research Council of Canada is gratefully acknowledged.

\section{REFERENCES}

[1] D. Mateescu, "Analysis of aerodynamic problems with geometrically unspecified boundaries using an enhanced Lagrangian method", J. Fluids. Struct.,vol. 17, pp. 603-626, 2003.

[2] C. Y. Loh, and W. H. Hui, "A new Lagrangian method for steady supersonic flow computation. Part 1 Godunov scheme", J. Comput. Phys., vol. 82, no.1, pp. 207-240, 1991.

[3] J. D. Anderson, Fundamentals of Aerodynamics. New York: McGraw-Hill, 2001.

[4] J. D. Anderson, Modern Compressible Flow With Historical Perspective. New York: McGraw-Hill, 2003.

[5] J. D. Anderson. Hypersonic and High Temperature Gas Dynamics. AIAA Education Series, 2006.

[6] M. A. Saad, Compressible Fluid Flow. New Jersey: Prentice-Hall, 1985.

[7] S. M. Yahya, Fundamentals of Compressible Flow. India: NewAge International, 2003.

[8] E. Carafoli, D. Mateescu and A. Nastase, Wing Theory in Supersonic Flow. New York: Pergamon Press, 1969. 